\title{
Estudo Experimental do Falar em Público Com e Sem Plateia em Universitários
}

\author{
Antonio Paulo Angélico' \\ Murilo Freitas Bauth ${ }^{1}$ \\ Arthur Kelles Andrade ${ }^{1}$ \\ ${ }^{1}$ Universidade Federal de São João del-Rei, São João del-Rei, $M G$
}

\begin{abstract}
Resumo
Objetivou-se comparar a frequência dos marcadores comportamentais de ansiedade entre grupos com e sem plateia, frente à situação experimental do falar em público. Participaram ao todo 72 universitários, que responderam ao Teste de Simulação de Falar em Público (TSFP), Inventário de Habilidades Sociais (IHS-Del-Prette), Self Statements During Public Speaking Scale (SPSS) e Questionário Sociodemográfico e Ocupacional. Os grupos diferiram significativamente em relação à maioria dos marcadores comportamentais de ansiedade, avaliados por meio do Protocolo de Registro do Falar em Público (PRFP), com médias de frequências superiores para o grupo com plateia. Constatou-se, ainda, que quanto mais elaborado o repertório de habilidades sociais de um universitário, mais positivamente ele avaliará o seu próprio desempenho em situações de falar em público. Sugerem-se novas pesquisas com maior número de universitários, com diferentes níveis de ansiedade social, que permitam examinar as associações entre habilidades sociais, autoavaliações ao falar em público e ansiedade social.
\end{abstract}

Palavras-chave: falar em público, avaliação do desempenho, interação social, autoavaliação

\begin{abstract}
Experimental Study of Public Speaking With and Without Audience in Undergraduates

This study aimed to compare the frequency of behavioral markers of anxiety between groups with and without an audience, against an experimental situation of public speaking. A total of 72 undergraduates participated, who responded to the Public Speaking Simulation Test, (TSFP), the Social Skills Inventory (IHS-Del-Prette), the Self Statements During Public Speaking Scale (SPSS), and the Sociodemographic and Occupational Questionnaire. The groups differed significantly considering most of the behavioral markers of anxiety, evaluated by the Public Speaking Register Protocol (PRFP), with higher frequency rates in the group with an audience. It was also found that the more elaborate is the social skills repertoire of an undergraduate, the more positive was his self-evaluation of his own performance in public speaking situations. It is suggested further research with a larger number of undergraduates with different levels of social anxiety, in order to allow the analysis of association between social skills, self-assessments when speaking in public, and social anxiety.

Keywords: public speaking, performance assessment, interpersonal relations, self-assessment.
\end{abstract}

\section{Resumen}

Estudio Experimental de Hablar en Público Con y Sin Audiencia en Estudiantes Universitarios

Se trató de comparar la frecuencia de los indicadores comportamentales de ansiedad entre los grupos participantes con y sin platea, frente a una situación experimental de hablar en público. Participaron 72 universitarios que respondieron Test de Simulación de Hablar en Público (TSFP) Inventario de Habilidades Sociales (IHS-Del-Prette), Self Statements During Public Speaking Scale (SPSS), y Cuestionario Sociodemográfico y Ocupacional. Los grupos diferían significativamente en relación a la mayoría de los indicadores comportamentales de ansiedad, evaluados por medio del Protocolo de Registro de Hablar en Público (PRFP), con promedio de frecuencia superior para el grupo con platea. Fue constatado, también, que cuanto más elaborado es el repertorio de habilidades sociales de un universitario, éste evaluará de forma más positiva su propio desempeño en situaciones de hablar en público. Se sugiere realizar nuevas investigaciones con un número mayor de universitarios, con diferentes niveles de ansiedad social, que permitan examinar las asociaciones entre habilidades sociales, autoevaluación al hablar en público y ansiedad social. Palabras-clave: hablar en público, evaluación de desempeño, interacción social, autoevaluación.

\section{Introdução}

O falar em público é um dos desempenhos sociais que mais exigem competência do indivíduo (Del Prette \& Del Prette, 2001a). Considerado um poderoso estressor psicossocial (Osório, Crippa, \& Loureiro, 2008), a tarefa de falar em público pode ser fonte de tremores, sudorese, gagueira, taquicardia, rubor, relaxamento dos esfíncteres, falhas na memória ("branco"), entre outras manifestações (Oliveira \& Duarte, 2004).
$\mathrm{Na}$ população universitária, problemas relacionados ao falar em público são bastante comuns. No estudo de Marinho, Medeiros, Gama e Teixeira (2017), com 1.135 estudantes universitários de uma universidade pública de Belo Horizonte (Minas Gerais), foi verificado que $63,9 \%$ da amostra reportou possuir medo de falar em público, sendo este medo mais prevalente entre mulheres $(72,5 \%)$, nos estudantes que participavam de poucas atividades de fala em grupo $(84,8 \%)$ e naqueles que autopercebiam suas vozes como 
negativas, com altura de voz para o agudo e volume de voz baixo $(68,7 \%)$. Em uma escala que examinava as autoavaliações frente à situação de falar em público (Self Statements During Public Speaking Scale - SSPS), foi observado que as mulheres apresentavam mais pensamentos negativos quando comparadas aos homens.

Estudantes universitários são, frequentemente, sujeitos a diversas exigências acadêmicas, como apresentações de trabalho, seminários em sala de aula e exposições de opinião em grupo, que podem eliciar altos níveis de ansiedade (Landim et al., 2000). A ansiedade excessiva, proveniente do comportamento de falar em público, pode ser causada por cognições negativas (Puteri \& Fakhurrozi, 2007); medo de possíveis consequências aversivas, resultantes de experiências anteriores; interpretações errôneas relacionadas à possíveis reações fisiológicas que serão experimentadas; e falta de familiaridade e preparo para a situação de falar em público (Clevenger \& Phifer, 1959).

Para Zimbardo (1982), os universitários que possuem medo de falar em público apresentam dificuldades em participar de seminários, não fazem perguntas para tirar dúvidas durante a aula e não são capazes de solicitar ajuda para o colega de classe. Segundo o referido autor, esses problemas podem afetar drasticamente o seu desempenho acadêmico, resultando em reprovações, dúvidas quanto à escolha profissional e desistência do curso universitário. $\mathrm{Na}$ tentativa de amenizar a ansiedade originada pelo desenvolvimento dessas tarefas, o medo de falar em público pode aumentar ainda o consumo de substâncias psicoativas, tais como álcool (Burke \& Stephens, 1999; Carrigan \& Randall, 2003; Gilles, Turk, \& Fresco, 2006) e diazepam (Chutuape \& Wit, 1995).

Em um estudo realizado por Puteri e Fakhurozzi (2007), foi avaliada a relação entre cognições positivas e a ansiedade frente à situação de falar em público em uma amostra de 50 estudantes universitários. Os resultados apontaram para fortes relações entre as variáveis citadas, indicando que indivíduos que mudam as suas cognições negativas, tornando-as positivas, apresentam melhoras significativas nos níveis de ansiedade frente à situação de falar em público.

Goberman, Hughes e Hydock (2011) investigaram os efeitos das características acústicas (fala e voz), autoavaliações e classificações da ansiedade por ouvintes em duas tarefas de falar em público com 16 universitários. Os dados levantados foram utilizados para análises posteriores que examinavam a ilusão de transparência, que consiste no fato do indivíduo pensar que sua ansiedade é mais perceptível aos ouvintes do que realmente é. Em outras palavras, trata-se de uma distorção da realidade, onde o indivíduo acha que seus estados privados, no caso a ansiedade, são facilmente acessíveis à plateia. Os resultados mostraram que, quando comparadas as avaliações dos participantes com as avaliações dos ouvintes, os indivíduos que discursaram se perceberam falando mais rápido, mais alto, com uma menor estabilidade na voz, mais ansiosos e tensos, e menos confiantes.

Nesse contexto, os estudantes universitários constituem uma classe emergente que, pelo menos em algumas áreas, possuem a interação social como base de sua ação profissional, seja como objeto ou objetivo dessa atuação (Del Prette, Del Prette, \& Castelo Branco, 1992a; Del Prette, Del Prette, \& Castelo Branco, 1992b). Por outro lado, considerando a alta prevalência do medo de falar em público, tanto na população geral (D’el Rey \& Pacini, 2005; Stein et al., 1996), quanto em universitários (Baptista, 2007; Marinho et al., 2017), com prejuízos sociais e funcionais evidenciados na qualidade de vida dos indivíduos, a necessidade de avaliação do repertório de habilidades sociais de falar em público e do referido medo desse grupo assume inquestionável relevância tanto social quanto educacional.

Muito se tem estudado os fatores relacionados ao falar em público em populações com transtorno de ansiedade social (Harb, Eng, Zairder, \& Heimberg, 2003; Laukka et al., 2008) e com altos níveis de ansiedade social (Kim, Lundh, \& Harvey, 2002; Schultz, Alpers, \& Hofmann, 2008). Outros estudos investigaram, ainda, as diferenças entre grupos com e sem o transtorno citado (Angélico, Crippa, \& Loureiro, 2012; Levitan et al., 2012; Rapee \& Abbott, 2007; Voncken \& Bögels, 2008) e com diferentes níveis de ansiedade social (Cody \& Teachman, 2011; Thompson \& Rapee, 2002; Wenzel \& Holt, 2003) na referida tarefa. Entretanto, verifica-se na literatura que pouco se tem investigado o medo de falar em público em populações sem o diagnóstico de algum tipo de transtorno psiquiátrico ou condições subclínicas (por exemplo, pessoas com altos níveis de ansiedade social, mas que não satisfazem os critérios diagnósticos do transtorno de ansiedade social).

A partir dessas considerações, o presente estudo teve como objetivo: (a) verificar as possíveis associações entre as manifestações subjetivas e comportamentais da ansiedade, apresentadas por estudantes universitários do curso de Psicologia frente a uma situação experimental de simulação do falar em público, denominada Teste de Simulação de Falar em Público (TSFP); e (b) verificar se os grupos com e sem 
plateia diferem em relação aos marcadores comportamentais de ansiedade e autoavaliações ao falar em público exibidos frente à situação experimental.

\section{Método}

\section{Participantes}

Participaram deste estudo 72 estudantes universitários, na faixa etária entre 17 e 30 anos $(M=21$ anos; $D P=2,73)$, pareados conforme o gênero, regularmente matriculados no curso de Psicologia de uma universidade pública do interior do estado de Minas Gerais, e designados em dois grupos, a saber: grupo com plateia $(n=32)$ e grupo sem plateia $(n=40)$. O grupo com plateia foi composto por 32 universitários (16 do gênero feminino e 16 do masculino), com idade média de 21,7 anos $(D P=2,90)$, variando de 18 a 30 anos, e 9,4\% exercendo alguma atividade profissional $(n=3)$. Em contrapartida, o grupo sem plateia foi composto por 40 universitários (20 do gênero feminino e 20 do masculino), com idade média 20,4 anos, variando de 17 a 27 anos, e $12,5 \%$ exercendo alguma atividade profissional $(n=5)$. Da amostra total, apenas $11,11 \%$ participantes trabalhavam e estudavam.

Esta pesquisa foi devidamente submetida à Comissão de Ética em Pesquisa envolvendo Seres Humanos da universidade para sua apreciação e, consequente, aprovação (Memo nº 067/2011-UFSJ/CEPES). Todos os universitários, que aceitaram participar do estudo, assinaram o Termo de Consentimento Livre e Esclarecido.

\section{Instrumentos e Materiais}

Neste estudo, foram empregados os seguintes instrumentos: Inventário de Habilidades Sociais (IHS-Del-Prette), Escala de Auto-Avaliação ao Falar em Público (Self Statements During Public Speaking Scale - SSPS), Protocolo de Registro do Falar em Público (PRFP), além da situação experimental do falar em público, denominada Teste de Simulação de Falar em Público (TSFP). Estes instrumentos serão descritos a seguir

Inventário de Habilidades Sociais (IHS-Del-Prette). É um instrumento de autorrelato, proposto por Del Prette e Del Prette (2001b), para a avaliação das dimensões situacional e comportamental molar das habilidades sociais, contendo 38 itens, cada um deles descrevendo uma relação interpessoal e uma possível reação àquela situação. Nas instruções, solicita-se que o respondente estime a frequência com que reage da forma sugerida em cada item, considerando o total de vezes em que se encontrou na situação descrita e anotando sua resposta em escala Likert, variando de nunca ou raramente a sempre ou quase sempre. Seguem descritos, abaixo, os dados iniciais de validade e confiabilidade do IHS-Del-Prette (Del Prette \& Del Prette, 2001b), obtidos por Del Prette, Del Prette e Barreto (1998) e Bandeira, Costa, Del Prette, Del Prette e Gerk-Carneiro (2000).

A análise das propriedades psicométricas, no estudo original, com universitários obteve os seguintes resultados: índices positivos de discriminação dos itens, variando de 3,0 a 16,7; índices positivos de correlação item-escore total, com apenas dois índices não significativos e 10 abaixo de 0,30 ; consistência interna satisfatória com um alfa de Cronbach de 0,75 ; e estrutura fatorial com cinco fatores principais, avaliada pelo método Alfa com rotação Varimax, cujos coeficientes alfa variaram de 0,74 a 0,96 , explicando $92,75 \%$ da variância total. Estes fatores foram denominados: enfrentamento e autoafirmação com risco (Fator 1), autoafirmação na expressão de sentimento positivo (Fator 2), conversação e desenvoltura social (Fator 3), autoexposição a desconhecidos e situações novas (Fator 4) e autocontrole da agressividade (Fator 5) (Del Prette et al., 1998). A fidedignidade foi avaliada por meio do método teste e reteste, obtendo-se um coeficiente de correlação de $0,90(p=0,001)$ (Bandeira et al., 2000).

\section{Self Statements During Public Speaking Scale} (SSPS). Instrumento autoaplicável, traduzido e adaptado para o português do Brasil por Osório et al., (2008), passando a chamar-se Escala para Autoavaliação ao Falar em Público. Esta escala visa mensurar a autopercepção do desempenho na situação específica de falar em público, composto por 10 itens, cada um deles descrevendo um pensamento que o respondente pode apresentar nesse tipo de circunstância. Em suas instruções, solicita-se que o respondente imagine as coisas que ele costuma pensar, sobre si mesmo, quando se encontra em alguma situação em que tenha que falar em público, considerando até que ponto o indivíduo concorda com as situações e anotando suas respostas em escala Likert, variando de 0 (discordo totalmente) a 5 (concordo inteiramente).

O instrumento possui qualidades psicométricas satisfatórias, em termos de fidedignidade e validade. A correlação dos itens com o escore total variou entre 0,44 e 0,71 . A validade de construto foi estudada por meio da análise da estrutura fatorial, realizada a partir da técnica de Análise dos Componentes Principais 
com rotação Varimax. Foram extraídos dois fatores que corresponderam conjuntamente $52 \%$ da variância dos dados (Osório, Crippa, \& Loureiro, 2012). A consistência interna apresentou um alfa de Cronbach de 0,90 para a escala total, 0,80 para a subescala de autoavaliação positiva (Fator 1) e 0,78 para a subescala de autoavaliação negativa (Fator 2) (Osório et al., 2008).

Teste de Simulação de Falar em Público (TSFP) (McNair et al., 1982). Consiste em solicitar ao sujeito que prepare um discurso e o apresente em frente a uma câmera de vídeo, que estará gravando seu desempenho. O modelo experimental do TSFP tem se mostrado capaz de induzir a ansiedade em voluntários saudáveis, independente do nível de traço de ansiedade, diferentemente de outros modelos, como o Stroop Color Word Test (Palma, Guimarães, \& Zuardi, 1994). As informações sobre a condução do TSFP são apresentadas na seção de "Procedimentos".

Protocolo de Registro do Falar em Público (PRFP, adaptado de Angélico, 2009). As categorias criadas para o protocolo de registro foram definidas e agrupadas em três classes de marcadores comportamentais de ansiedade, a saber: (a) marcadores verbais (por exemplo, conteúdo negativo e uso de jargões); (b) marcadores paralinguísticos (pausas silenciosas e velocidade de fala inapropriada); e (c) marcadores não verbais, que compreendem as posturas corporais e gestos apresentados pelos participantes (inquietação e barreiras corporais). Para a observação dos marcadores verbais e paralinguísticos, foi empregada a técnica de registro de evento por fração de minuto, com intervalos de 30 segundos; e para a observação dos marcadores não verbais, a técnica de registro a intervalos de 15 segundos.

Visando a consecução dos objetivos desta pesquisa, além desses instrumentos, foram utilizados os seguintes equipamentos e materiais durante o período de coleta de dados: uma câmara digital, uma televisão, um computador e DVDs. Para a análise dos dados, foram empregados: televisão, DVD Player, computador, DVDs com as filmagens do TSFP, e PRFP.

Para esta última subescala, a pontuação dos itens é invertida, ou seja, quanto menor a média da pontuação, maior a avaliação negativa que o indivíduo tem de si (Osório et al., 2008).

\section{Procedimentos}

Oito assistentes, previamente treinados, compuseram a equipe desta pesquisa. A coleta de dados foi realizada em duas etapas, a primeira com os participantes do grupo com plateia a segunda com os integrantes do grupo sem plateia. Em cada etapa, um assistente de pesquisa conduziu o TSFP e outro, a aplicação das demais escalas. O conteúdo das filmagens foi analisado pelos assistentes de pesquisa, visando o cálculo das frequências ou duração dos marcadores comportamentais de ansiedade e dos índices de concordância entre observadores, a partir da seguinte fórmula:

$$
\begin{gathered}
\text { Índice de } \\
\text { Concordância }(\mathrm{IC})
\end{gathered}=\frac{\text { concordâncias }}{\text { concordâncias }+ \text { discordâncias }} \times 100 \text { ” }
$$

Aos participantes, foi solicitado comparecerem no Laboratório de Ensino de Psicologia Experimental (LAPEX) da universidade, em dia e horário previamente agendado, de acordo com a disponibilidade individual, para participarem de uma situação experimental considerada um teste de ansiedade. $\mathrm{Na}$ ocasião da aplicação do teste, primeiramente, foram explicados os objetivos e procedimentos do estudo ao participante. Em seguida, entregavam-lhe o Termo de Consentimento Livre e Esclarecido (TCLE) para que lesse e assinasse. Somente prosseguiram aqueles participantes que devidamente assinaram o TCLE.

Posteriormente, os sujeitos preencheram o Questionário Sociodemográfico, IHS-Del-Prette e SSPS, sendo este respondido antes e após a tarefa de falar em público. O procedimento completo de coleta de dados durou aproximadamente uma hora.

Com relação ao TSFP, o participante ficava em frente a uma câmara de vídeo e assistia a um videotape pré-gravado com instruções sobre a tarefa que teria que desempenhar. Recebia a seguinte instrução: "Você terá dois minutos para preparar um discurso, de quatro minutos sobre sistema de cotas na universidade, que será gravado e, posteriormente, analisado por um psicólogo". Era computado o tempo de dois minutos para a preparação do discurso e, ao término, solicitado ao participante que o iniciasse. Transcorrido os quatro minutos, solicitava-se que permanecesse no ambiente, procurando relaxar, encerrando-se a sessão experimental.

Os procedimentos listados acima foram aplicados em duas fases distintas, descritas a seguir. Em uma primeira fase do estudo, os participantes, designados para o grupo com plateia, proferiram o discurso para uma audiência composta por quatro expectadores (assistentes de pesquisa). $\mathrm{Na}$ segunda fase, enquanto os participantes (grupo sem plateia) proferiam seu discurso frente à câmera, seus desempenhos eram simultaneamente transmitidos na tela da televisão, posicionada 
também a sua frente, para que pudessem acompanhar as suas performances e, assim, aumentar a veracidade da situação, de acordo McNair et al. (1982).

\section{Análise de Dados}

Os dados obtidos para a análise do conteúdo das filmagens foram codificados manualmente e alocados em um banco de dados criado em uma planilha do pacote estatístico SPSS (Statistical Package for Social Sciences) para o Windows, versão 22.0. Em seguida, análises estatísticas foram conduzidas com esses dados, por meio dos testes descritivos de média e desvios-padrão. Com exceção dos escores totais e fatoriais do IHS-Del-Prette, os dados relativos às demais 26 variáveis testadas demonstraram não seguir distribuição normal $(p<0,05)$. Sendo assim, foram empregados testes não paramétricos para todas as análises estatísticas deste estudo. Para todos os testes estatísticos, foi adotado o nível de significância de $p<0,05$.

O procedimento de categorização e análise das sessões experimentais seguiu os passos relatados a seguir. A partir das filmagens de cada participante: (a) verificaram-se as ocorrências dos marcadores comportamentais, denominados de categorias, registrando-se as suas frequências; e (b) computaram-se a duração da latência para o participante iniciar o discurso. Todas essas ações foram realizadas utilizando-se o protocolo de registro. O conteúdo do discurso dos participantes foi transcrito para possibilitar análises e categorizações mais precisas dos marcadores verbais de ansiedade.

Para verificar o efeito do tempo sobre os marcadores comportamentais de ansiedade e suas respectivas classes ao longo do TSFP, comparando-se os grupos com e sem plateia, foi realizada uma análise de variância de medidas repetidas de um fator (ANOVA oneway). Para tal, selecionaram-se os dados dos dois primeiros (M1) e dos dois últimos minutos (M2) do discurso. Quando a diferença entre estes dois momentos não foi significativamente a mesma para ambos os grupos, foi realizado testes de Wilcoxon para os grupos separadamente com o intuito de identificar o grupo que apresentou a diferença.

Empregou-se o teste de Mann-Whitney para comparar os grupos com e sem plateia em relação aos marcadores de ansiedade e suas respectivas classes durante o TSFP. Para análises intragrupos, utilizou-se o teste de Wilcoxon. Esses mesmos procedimentos estatísticos foram conduzidos para as autoavaliações ao falar em público, antes e após a situação experimental. Análises de correlação bivariada de Spearman foram realizadas para investigar as relações entre os escores totais e fatoriais do IHS-Del-Prette, as subescalas positivas e negativas do SSPS, os itens e escore geral das habilidades sociais de falar em público (HSFP), extraídos do IHS, e os marcadores de ansiedade e suas respectivas classes.

Para presente análise, foram selecionados os itens do IHS-Del-Prette que melhor se ajustassem à habilidade de falar em público, a saber: itens 9 ("Evito fazer exposições ou palestras a pessoas desconhecidas"), 11 ("Em uma sala de aula ou reunião, se o professor ou dirigente faz uma afirmação incorreta, eu expresso meu ponto de vista"), 14 ("Faço exposição (por exemplo, palestras) em sala de aula ou no trabalho, quando sou indicado(a)"), 16 ("Em um grupo de pessoas conhecidas, se não concordo com a maioria, expresso verbalmente minha discordância") e 29 ("Na escola ou no trabalho, quando não compreendo uma explicação sobre algo que estou interessado(a), faço as perguntas que julgo necessárias ao meu esclarecimento"). Estes itens tiveram em comum o componente de falar e/ ou se expor em público, alguns deles com possibilidade de rejeição, réplica, ou de oposição por parte do(s) interlocutor(es). Nas análises conduzidas, estes itens foram também somados para se obter um escore geral referente à habilidade de falar em público, sendo consideradas apenas as correlações que demostraram significância estatística e sentido de conteúdo.

No tocante à avaliação da ocorrência dos marcadores comportamentais de ansiedade e suas respectivas classes durante a tarefa de falar em público, foram computados índices de concordância (ICs) entre duplas de observadores, formadas entre cinco assistentes de pesquisa, previamente treinados. Para os marcadores de ansiedade, os índices obtidos variaram de 96,8 (repetição de conteúdo) a 99,9\% (evitação da tarefa). Considerando as classes de marcadores, os índices variaram de 98,2 (classe de marcadores verbais) a 99,5\% (classe de marcadores não verbais). No geral, obteve-se um IC de 99,0\% entre os observadores. Caso os observadores obtivessem ICs inferiores a $85 \%$, assistiam e analisavam o desempenho do participante em questão, conjuntamente, para identificar e discutir as possíveis divergências, visando chegar a um consenso sobre a ocorrência e frequência de marcadores específicos.

\section{Resultados}

Uma ANOVA foi conduzida para comparar o efeito de tempo sobre os marcadores comportamentais 
Tabela 1

Comparação do Efeito do Tempo sobre os Marcadores Comportamentais de Ansiedade e suas Respectivas Classes e de sua Interação com os Grupos Com Plateia $(n=32)$ e Sem Plateia $(n=40)$

\begin{tabular}{|c|c|c|c|c|}
\hline \multirow{2}{*}{$\begin{array}{l}\text { Diferença entre M1 e M2 em relação aos } \\
\text { marcadores comportamentais e suas classes }\end{array}$} & \multicolumn{2}{|c|}{ Efeito Global } & \multicolumn{2}{|c|}{ Efeito para Grupos } \\
\hline & $F$ & Probabilidade & $F$ & Probabilidade \\
\hline Conteúdo negativo & 35,94 & $p<0,001^{*}$ & 1,22 & $p=0,273^{(\mathrm{NS})}$ \\
\hline Repetição de conteúdo & 0,97 & $p=0,328^{(\mathrm{NS})}$ & 0,74 & $p=0,394^{(\mathrm{NS})}$ \\
\hline Uso de jargões & 11,31 & $p=0,001 *$ & 0,07 & $p=0,795^{(\mathrm{NS})}$ \\
\hline Repetição de palavra & 11,10 & $p=0,001^{*}$ & 0,81 & $p=0,371^{(\mathrm{NS})}$ \\
\hline Pausas silenciosas & 0,27 & $p=0,607^{(\mathrm{NS})}$ & 2,31 & $p=0,133^{(\mathrm{NS})}$ \\
\hline Pausas preenchidas & 48,59 & $p<0,001 *$ & 0,81 & $p=0,370^{(\mathrm{NS})}$ \\
\hline Velocidade de fala inapropriada & 20,22 & $p<0,001 *$ & 0,52 & $p=0,474^{(\mathrm{NS})}$ \\
\hline Intensidade de voz inapropriada & 1,22 & $p=0,272^{(\mathrm{NS})}$ & 0,001 & $p=0,982^{(\mathrm{NS})}$ \\
\hline Evitação de tarefa & 70,92 & $p<0,001 *$ & 2,42 & $p=0,125^{(\mathrm{NS})}$ \\
\hline Rigidez corporal & 6,41 & $p=0,014^{*}$ & 34,97 & $p<0,001 *$ \\
\hline Inquietação & 2,88 & $p=0,094^{(\mathrm{NS})}$ & 8,48 & $p=0,005^{*}$ \\
\hline Barreiras & 12,18 & $p=0,001 *$ & 20,81 & $p<0,001^{*}$ \\
\hline Classe de marcadores verbais & 27,68 & $p<0,001^{*}$ & 0,87 & $p=0,355^{(\mathrm{NS})}$ \\
\hline Classe de marcadores paralinguísticos & 34,68 & $p<0,001 *$ & 0,43 & $p=0,514^{(\mathrm{NS})}$ \\
\hline Classe de marcadores não verbais & 0,46 & $p=0,501^{(\mathrm{NS})}$ & 33,83 & $p<0,001^{*}$ \\
\hline
\end{tabular}

Nota. M1 = Momento 1; M2 = Momento 2; F = F de Fisher; $*$ = diferença significativa; $(\mathrm{NS})=$ diferença não significativa.

de ansiedade e suas respectivas classes e em relação aos grupos com e sem plateia. Os resultados obtidos encontram-se na Tabela 1.

Quanto ao efeito global da tarefa, observaram-se diferenças significativas entre os dois primeiros minutos (M1) e os dois últimos minutos (M2) do discurso para conteúdo negativo, uso de jargões, repetição de palavra, pausas preenchidas, velocidade de fala inapropriada, rigidez corporal, evitação de tarefa, barreiras, classes de marcadores verbais e paralinguísticos. $\mathrm{Na}$ comparação dos grupos com e sem plateia, verificou-se que essa diferença foi significativa para rigidez corporal, inquietação, barreiras e classe de marcadores não verbais. Para o grupo com plateia, a diferença entre M1 e M2 foi significativa apenas para rigidez corporal e classe de marcadores não verbais $(Z=-2,66 ; p=0,008$ e $Z$ $=3,84 ; p<0,001$, respectivamente), com aumento $\mathrm{da}$ frequência de ambas as categorias para os dois últimos minutos do discurso. Em contrapartida, no grupo sem plateia, a diferença foi significativa para rigidez corporal $(Z=-4,19 ; p<0,001)$, inquietação $(Z=-3,26 ; p$ $=0,001)$, barreiras $(Z=-3,75 ; p<0,001)$ e classe de marcadores não verbais $(Z=-3,44 ; p=0,001)$, com diminuição da frequência durante os dois últimos minutos do discurso para todas essas categorias.

A Tabela 2 exibe os dados relativos à comparação da frequência de cada um dos marcadores comportamentais de ansiedade e suas respectivas classes, durante o TSFP, entre os grupos com e sem plateia. Nesta tabela, estão dispostos os valores da média e desvio-padrão dos marcadores e classes para cada grupo e do teste de Mann-Whitney.

No geral, os grupos diferiram significativamente em relação à maioria das categorias e classes avaliadas: latência de resposta, conteúdo negativo, repetição de conteúdo, pausas silenciosas, intensidade de voz inapropriada, rigidez corporal, inquietação, barreiras, classes de marcadores paralinguísticos e não verbais. Com exceção de latência de resposta e conteúdo negativo, o grupo com plateia exibiu uma média de frequência significativamente mais alta para todas as demais categorias e classes. Embora as diferenças de médias para uso de jargões, pausas preenchidas, velocidade de fala inapropriada e classe de marcadores 
Tabela 2

Comparação dos Grupos Com Plateia $(n=32)$ e Sem Plateia $(n=40)$ em relação aos Marcadores Comportamentais de Ansiedade e suas Respectivas Classes

\begin{tabular}{|c|c|c|c|c|}
\hline \multirow{2}{*}{ Categorias e Classes Avaliadas } & Com Plateia & Sem Plateia & \multirow{2}{*}{$\begin{array}{c}U \text { de Mann- } \\
\text { Whitney }\end{array}$} & \multirow{2}{*}{ Probabilidade } \\
\hline & Média $(D P)$ & Média $(D P)$ & & \\
\hline Latência de resposta & $0,31(1,06)$ & $1,03(1,21)$ & 341,0 & $p<0,001 *$ \\
\hline Conteúdo negativo & $4,25(2,65)$ & $5,85(3,53)$ & 452,0 & $p=0,031 *$ \\
\hline Repetição de conteúdo & $4,16(3,10)$ & $2,20(1,84)$ & 372,0 & $p=0,002 *$ \\
\hline Uso de jargões & $6,91(6,43)$ & $4,03(4,04)$ & 494,5 & $p=0,097^{(\mathrm{NS})}$ \\
\hline Repetição de palavra & $7,19(7,41)$ & $7,65(6,20)$ & 585,5 & $p=0,536^{(\mathrm{NS})}$ \\
\hline Pausas silenciosas & $2,16(2,80)$ & $1,20(2,36)$ & 465,5 & $p=0,035^{*}$ \\
\hline Pausas preenchidas & $6,44(4,91)$ & $5,45(4,77)$ & 565,5 & $p=0,395^{(\mathrm{NS})}$ \\
\hline Velocidade de fala inapropriada & $5,03(4,78)$ & $3,18(3,77)$ & 486,5 & $p=0,078^{(\mathrm{NS})}$ \\
\hline Intensidade de voz inapropriada & $5,31(4,18)$ & $1,45(1,47)$ & 240,5 & $p<0,001 *$ \\
\hline Evitação de tarefa & $3,88(4,49)$ & $4,73(4,15)$ & 575,0 & $p=0,451^{(\mathrm{NS})}$ \\
\hline Rigidez corporal & $12,03(4,60)$ & $7,98(4,93)$ & 350,0 & $p=0,001^{*}$ \\
\hline Inquietação & $15,56(8,82)$ & $5,00(5,26)$ & 195,5 & $p<0,001 *$ \\
\hline Barreiras & $22,75(8,81)$ & $15,40(7,86)$ & 345,0 & $p=0,001 *$ \\
\hline Classe de marcadores verbais & $22,50(14,80)$ & $19,75(11,02)$ & 589,5 & $p=0,567^{(\mathrm{NS})}$ \\
\hline Classe de marcadores paralinguísticos & $18,94(10,52)$ & $10,87(6,78)$ & 336,0 & $p=0,001 *$ \\
\hline Classe de marcadores não verbais & $54,22(17,05)$ & $32,78(11,51)$ & 217,5 & $p<0,001^{*}$ \\
\hline
\end{tabular}

Nota. DP = desvio padrão; $*$ diferença significativa; $(\mathrm{NS})=$ diferença não significativa.

verbais não foram estatisticamente significativas, o grupo com plateia apresentou valores maiores em comparação ao grupo sem plateia.

A Tabela 3 apresenta os dados referentes à comparação entre os grupos com e sem plateia quanto às autoavaliações ao falar em público antes e depois da situação experimental. Para as análises, foram empregadas as subescalas positiva e negativa da SSPS.

Foram verificadas diferenças estatisticamente significativas entre os grupos experimentais (com e sem plateia), apenas, no primeiro momento (M1) para as autoavaliações positivas $(p=0,034)$ e negativas $(p=$ $0,010)$. Para ambas as subescalas, o grupo sem plateia obteve pontuações médias significativamente superiores, comparativamente ao grupo com plateia. Apesar dos grupos não atestarem diferenças significativas no segundo momento (M2), o grupo sem plateia apresentou valores ligeiramente superiores para as duas subescalas em relação ao grupo com plateia. Considerando os grupos com e sem plateia, separadamente, as diferenças entre as subescalas positiva e negativa, para M1, não foram significas $(Z=-0,69 ; p=0,490$ e $Z=$
$-1,85 ; p=0,065$, respectivamente), com maiores pontuações para a subescala negativa. Ambos os grupos, separadamente, também não demonstraram diferenças significativas nas pontuações das subescalas entre M1 e M2 $(p \leq 0,095)$.

Os dados referentes às correlações entre os escores totais e fatoriais do IHS-Del-Prette, os itens relativos às habilidades sociais de falar em público e o seu escore geral extraídos deste mesmo instrumento de medida, as subescalas positiva e negativa do SPSS, e os marcadores comportamentais de ansiedade e suas respectivas classes estão expostos na Tabela 4. Nesta tabela, estão contidos os coeficientes de correlação de Spearman obtidos e os valores de $p$ correspondentes.

Verificou-se que o escore total, os itens 9, 29, o escore geral de HSFP, e os Fatores 1, 2, 3 e 4 do IHS-Del-Prette se correlacionaram positiva e significativamente com a subescala positiva e negativa SSPS. Além disso, a subescala negativa da SSPS correlacionou positiva e significativamente com os itens $11 \mathrm{e}$ 14 do IHS. No que se refere às categorias de análise do TSFP, foi constatado que latência de fala se 
Tabela 3

Comparação dos Grupos Com Plateia $(n=32)$ e Sem Plateia $(n=40)$ em relação às Autoavaliações ao Falar em Público Antes e Após a Tarefa de Falar em Público

\begin{tabular}{|c|c|c|c|c|}
\hline \multirow{2}{*}{$\begin{array}{l}\text { Self Statements During Public } \\
\text { Speaking Scale }\end{array}$} & \multirow{2}{*}{$\begin{array}{l}\text { Com Plateia } \\
\text { Média }(D P)\end{array}$} & \multirow{2}{*}{$\begin{array}{c}\text { Sem Plateia } \\
\text { Média }(D P)\end{array}$} & \multirow{2}{*}{$\begin{array}{l}\text { U de Mann- } \\
\text { Whitney }\end{array}$} & \multirow{2}{*}{ Probabilidade } \\
\hline & & & & \\
\hline SSPS-P (M1) & $17,13(4,09)$ & $19,00(3,06)$ & 454,0 & $p=0,034^{*}$ \\
\hline SSPS-N (M1) & $17,43(6,16)$ & $20,59(4,37)$ & 414,5 & $p=0,010^{*}$ \\
\hline SSPS-P (M2) & $17,85(4,60)$ & $18,56(4,30)$ & 582,5 & $p=0,512^{(\mathrm{NS})}$ \\
\hline SSPS-N (M2) & $17,88(6,10)$ & $19,50(5,77)$ & 534,0 & $p=0,227^{(\mathrm{NS})}$ \\
\hline
\end{tabular}

Nota. SSPS-P = Subescala de autoavaliação positiva; SSPS-N = Subescala de autoavaliação negativa; M1 = Momento 1; M2 = Momento 2; DP = desvio padrão; $*$ = diferença significativa; $(\mathrm{NS})=$ diferença não significativa.

Tabela 4

Correlações Significativas Encontradas entre Habilidades Sociais Gerais e de Falar em Público, Autoavaliações e Marcadores Comportamentais de Ansiedade na Amostra do Estudo (N = 72)

\begin{tabular}{lcc}
\hline Correlações significativas & $\rho$ de Spearman & Probabilidade \\
\hline Escore total IHS x subescala positiva SSPS & 0,46 & $p<0,001$ \\
Escore total IHS x subescala negativa SSPS & 0,56 & $p<0,001$ \\
Fator 1 IHS x subescala positiva SSPS & 0,33 & $p=0,005$ \\
Fator 1 IHS x subescala negativa SSPS & 0,46 & $p<0,001$ \\
Fator 2 IHS x subescala positiva SSPS & 0,32 & $p=0,006$ \\
Fator 2 IHS x subescala negativa SSPS & 0,33 & $p=0,005$ \\
Fator 3 IHS x subescala positiva SSPS & 0,38 & $p=0,001$ \\
Fator 3 IHS x subescala negativa SSPS & 0,44 & $p<0,001$ \\
Fator 3 IHS x latência da resposta & $-0,24$ & $p=0,042$ \\
Fator 4 IHS x subescala positiva SSPS & 0,38 & $p=0,001$ \\
Fator 4 IHS x subescala negativa SSPS & 0,43 & $p<0,001$ \\
Fator 5 IHS x repetição de conteúdo & $-0,27$ & $p=0,021$ \\
Item 9 IHS (Falar a público desconhecido) x subescala positiva SSPS & 0,37 & $p=0,002$ \\
Item 9 IHS (Falar a público desconhecido) x subescala negativa SSPS & 0,48 & $p<0,001$ \\
Item 9 IHS (Falar a público desconhecido) x evitação de tarefa & $-0,38$ & $p=0,001$ \\
Item 11 IHS (Discordar de autoridade) x subescala negativa SSPS & 0,35 & $p=0,002$ \\
Item 14 IHS (Falar a público conhecido) x subescala negativa SSPS & 0,38 & $p=0,001$ \\
Item 29 IHS (Fazer pergunta a conhecidos) x subescala positiva SSPS & 0,31 & $p=0,008$ \\
Item 29 IHS (Fazer pergunta a conhecidos) x subescala negativa SSPS & 0,52 & $p<0,001$ \\
Item 29 IHS (Fazer pergunta a conhecidos) x evitação de tarefa & $-0,31$ & $p=0,008$ \\
Escore geral HSFP x subescala positiva SSPS & 0,31 & $p=0,008$ \\
Escore geral HSFP x subescala negativa SSPS & 0,54 & $p<0,001$ \\
Escore geral HSFP x evitação de tarefa & $-0,35$ & $p=0,002$ \\
Subescala positiva SSPS x latência da resposta & $-0,24$ & $p=0,042$ \\
\hline
\end{tabular}

Nota. IHS: Inventário de Habilidades Sociais; Fator 1: Enfrentamento com risco; Fator 2: Autoafirmação na expressão de afeto positivo; Fator 3: Conversação e desenvoltura social; Fator 4: Autoexposição a desconhecidos ou a situações novas; Fator 5: Autocontrole da agressividade em situações aversivas; HSFP: Habilidades Sociais de Falar em Público; SSPS: Self Statements During Public Speaking Scale. 
correlacionou negativa e significativamente com o Fator 3 do IHS e com a subescala positiva do SSPS. Para a categoria evitação de tarefa, foram encontradas correlações negativas e significativas com o item 9, 29 e o escore geral da HSFP. A categoria repetição de conteúdo se correlacionou negativa e significativamente com o Fator 5 do IHS.

\section{Discussão}

No que diz respeito ao efeito global da tarefa de falar em público sobre o desempenho dos participantes, foi observado que, com exceção de evitação de tarefa, a frequência de emissão da maioria das classes e marcadores comportamentais de ansiedade diminuiu significativamente entre os dois primeiros e os dois últimos minutos do TSFP, apontando para a ocorrência de uma dessensibilização comportamental da ansiedade ao longo do teste. Esses resultados corroboram aqueles encontrados por Goberman et al. (2011), que verificaram que, no decurso de tarefas de falar em público, os universitários apresentaram maiores níveis de ansiedade no início dos discursos, comparativamente ao seu final, havendo, assim, a dessensibilização do efeito ansiogênico. Segundo os autores, essa diminuição pode ter ocorrido também pelo fato dos participantes se sentirem mais confortáveis com o fim da tarefa se aproximando.

Na comparação entre os grupos (com e sem plateia), constatou-se que a dessensibilização do efeito ansiogênico da tarefa de falar em público não ocorreu para o grupo com plateia. Pode-se sugerir duas hipóteses para esses resultados: a dessensibilização não ocorreu devido à (a) duração curta e (b) maior complexidade da tarefa. Embora utilizasse marcadores de ansiedade distintos, o estudo de Goberman et al. (2011) pode reforçar essa hipótese, visto que apresentava uma tarefa com uma duração maior, propiciando um tempo mais prolongado para a dessensibilização do efeito ansiogênico ocorrer. O efeito ansiogênico da tarefa foi menos acentuado para o grupo sem plateia, que não contava com uma audiência real de expectadores assistindo ao seu discurso. De acordo com Angélico et al. (2012), isso pode ocorrer também devido a um bom processamento cognitivo para alguns estímulos externos dos participantes, que eram capazes de discriminar que estavam se comportando frente a uma câmera e não a uma audiência de pessoas.

Com relação às autoavaliações do falar em público, pode-se afirmar que os resultados encontrados foram consistentes com as hipóteses subjacentes do presente estudo, pois o grupo sem plateia apresentou autoavaliações significativamente mais positivas em comparação ao grupo com plateia para o primeiro momento do TSFP. Em acréscimo, os grupos com e sem plateia não demonstraram diferenças significativas entre os valores obtidos para as subescalas positiva e negativa, ou seja, não se constatou predominância de um tipo específico de autoavaliação frente à situação de falar em público, sugerindo apenas uma tendência para autoavaliações negativas.

Quanto às correlações significativas obtidas neste estudo, constatou-se que: (a) quanto mais elaborado o repertório global de habilidades sociais de uma pessoa e, em especial, das classes de enfrentamento com risco, autoafirmação na expressão de afeto positivo, conversação e desenvoltura social, autoexposição a desconhecidos ou a situações novas, e de falar em público, mais positivamente a pessoa avaliou o seu próprio desempenho frente à situação de falar em público; (b) quanto mais desenvolvida a classe de habilidades de conversação e desenvoltura social, e mais positivas fossem as autoavaliações de um indivíduo, menor era a sua latência de resposta em situações de falar em público; (c) quanto mais ricas as habilidades de falar em público de uma pessoa, menor foi a sua evitação de tarefa de fazer discursos; e (d) quanto mais rica a classe de habilidades de autocontrole da agressividade em situações aversivas de um indivíduo, menor será a sua frequência de repetir conteúdo. Diante do exposto, constata-se uma relação direta existente entre habilidades sociais, autoavaliações ( $\operatorname{cog}$ nições) e alguns marcadores críticos de ansiedade frente à tarefa de falar em público. Pode-se, assim, afirmar que quanto mais elaborado for o repertório de habilidades sociais gerais e de falar em público, apresentado por um indivíduo, mais positivas serão as suas autoavaliações (cognições) diante de tarefas de falar em público e, por sua vez, menos frequente será a sua exibição de marcadores comportamentais de ansiedade.

Em contrapartida, como defendido por Lopez (1986), a ausência das habilidades necessárias para falar em público (déficit de habilidades) e o modo inadequado com que a pessoa avalia as situações em que essas habilidades são requeridas (avaliação cognitiva falha) podem interferir na performance adequada em situações de falar em público. Esta avaliação cognitiva pode se referir tanto ao próprio desempenho da pessoa quanto às expectativas de consequências aversivas frente a tarefas de falar em público (por exemplo, desaprovação dos demais). 
Para Del Prette e Del Prette (2001), além do controle da ansiedade, a habilidade de falar em público abarca ainda outros dois componentes; a saber: (a) cognitivos relacionados ao conhecimento das características do público, domínio do conteúdo a ser transmitido e a preparação dos recursos da apresentação; e (b) metacognitivos que dizem respeito ao automonitoramento durante uma apresentação e à previsão de estratégias para lidar com possíveis reações dos ouvintes. Esses componentes não foram avaliados pelo presente estudo, podendo ser explorados em pesquisas futuras.

No que diz respeito ao domínio de conteúdo, o presente estudo ofereceu apenas uma opção de tema para o discurso ("sistema de cotas na universidade"), o que pode ter influenciado negativamente o desempenho dos participantes. No estudo de McNair et al. (1982), por exemplo, os sujeitos podiam escolher um tema dentre cinco tópicos neutros selecionados randomicamente de um conjunto 54 possibilidades. Em outro estudo, estudantes de biomedicina escolhiam aleatoriamente um tema lista de 17 tópicos sobre fisiologia (Guimarães, Zuardi, \& Graeff, 1987). Já Goberman et al. (2010), instruíram os participantes a prepararem dois discursos. No primeiro, eles deviam falar sobre si mesmos e no segundo sobre qualquer outra temática.

De modo geral, os estudos encontrados na literatura sobre os fatores relacionados ao falar em público, como já citado anteriormente, estão voltados mais para populações com transtorno de ansiedade social (Harb, Eng, Zairder, \& Heimberg, 2003; Laukka et al., 2008), com altos níveis de ansiedade social (Kim, Lundh, \& Harvey, 2002; Schultz, Alpers, \& Hofmann, 2008) e, também, para comparações entre grupos com e sem o transtorno mencionado (Angélico, Crippa, \& Loureiro, 2012; Levitan et al., 2012; Rapee \& Abbott, 2007; Voncken \& Bögels, 2008) e com diferentes níveis de ansiedade social (Cody \& Teachman, 2011; Thompson \& Rapee, 2002; Wenzel \& Holt, 2003). Em contrapartida, a amostra deste estudo foi composta apenas por universitários que não foram submetidos à avaliação clínica, segundo critérios diagnósticos, para transtornos psiquiátricos ou quaisquer condições subclínicas, impossibilitando, assim, que seus dados fossem comparados com aqueles obtidos nas demais pesquisas da área.

\section{Conclusões}

Em geral, foram confirmadas as associações entre as manifestações subjetivas (autoavaliações ao falar em público) e comportamentais da ansiedade apresentadas pelos estudantes universitários frente a uma situação experimental de simulação do falar em público. Ademais, como esperado, os grupos com e sem plateia diferiram significativamente em relação à maioria dos marcadores comportamentais de ansiedade, com médias de frequências superiores para o primeiro grupo.

De acordo com Angélico et al. (2012), o TSFP apresenta-se como uma forma confiável e útil para a avaliação de marcadores comportamentais da ansiedade. As medidas objetivas de ansiedade, obtidas ao longo do teste, forneceram informações importantes relacionadas aos pontos fracos específicos dos indivíduos avaliados, que podem ser usadas tanto para o planejamento quanto no curso de programas de Treinamento de Habilidades Sociais (THS), com o objetivo de reduzir déficits e promover um melhor desempenho em tarefas de falar em público.

A partir dos resultados encontrados, verifica-se a possibilidade de uso do TSFP no planejamento de programas de THS voltados para a população de universitários, dada a alta prevalência do medo de falar em público. Mais especificamente, os dados obtidos no TSFP podem ser empregados em programas de THS no contexto educacional para a avaliação tanto dos recursos quanto dos déficits comportamentais de habilidades sociais de falar em público, visando promover um melhor desempenho de universitários nas atividades acadêmicas de apresentação de trabalhos, seminários e participação em congressos científicos. Em acréscimo, o refinamento no repertório comportamental dos alunos refletiria, futuramente, em uma melhor qualificação profissional dos mesmos.

Entretanto, esse estudo apresentou algumas limitações quanto aos procedimentos metodológicos empregados. Além de não ter sido conduzida uma análise de equivalência dos grupos quanto ao repertório de habilidades sociais, essa pesquisa possuiu um número reduzido de sujeitos em cada grupo experimental e foi realizada apenas com um curso de graduação, impossibilitando a generalização dos resultados para outros cursos. Desta forma, recomenda-se a realização de novas pesquisas com maior número de universitários, designados randomicamente aos grupos com níveis de ansiedade social alto e baixo, de diferentes cursos e áreas acadêmicas, que permitam a generalização dos dados obtidos e o exame da associação entre habilidades sociais, autoavaliações ao falar em público e ansiedade social. Além disso, tendo em vista que os participantes deste estudo não puderam escolher um tema 
que dominassem para o discurso, sugere-se que futuras pesquisas proporcionem uma maior autonomia aos indivíduos na seleção de temas para a tarefa de falar em público, seja por meio de um número maior de opções ou por livre escolha.

\section{Referências}

Angélico, A. P. (2009). Transtorno de ansiedade social e habilidades sociais: Estudo psicométrico e empírico (Tese de doutorado). Universidade de São Paulo, Ribeirão Preto.

Angélico, A. P., Crippa, J. A. S., \& Loureiro, S. R. (2012). Transtorno de ansiedade social e habilidades sociais de falar em público: Estudo experimental. Estudos e Pesquisas em Psicologia, 12(1), 10-35. Recuperado de http://pepsic.bvsalud.org/pdf/epp/ v12n1/v12n1a02.pdf

Bandeira, M., Costa, M. N., Del Prette Z. A. P., Del Prette, A., \& Gerk-Carneiro, E. (2000). Qualidades psicométricas do Inventário de Habilidades Sociais (IHS): Estudo sobre a estabilidade temporal e validade concomitante. Estudos de Psicologia (Natal), 5(2), 401-419. doi: 10.1590/S1413-294X2000000200006

Baptista, C. A. (2007). Estudo da prevalência do transtorno de ansiedade social em estudantes universitários (Dissertação de mestrado). Faculdade de Medicina de Ribeirão Preto, Universidade de São Paulo, São Paulo. Recuperado de http://pgsm.fmrp.usp.br/wp-content/ uploads/2011/11/MESTRADO-CARLOS-ALBERTO-BAPTISTA.pdf

Burke, R. S., \& Stephens, R. S. (1999). Social anxiety and drinking in college students: A social cognitive theory analysis. Clinical Psychology Review, 19(5), 513530. doi: 10.1016/S0272-7358(98)00058-0

Carrigan, M. H., \& Randall, C. L. (2003). Self-medication in social phobia: A review of the alcohol literature. Addictive Behaviors, 28(2), 269-284. doi: 10.1016/S0306-4603(01)00235-0

Chutuape, M. A., \& de Wit, H. (1995). Preferences for ethanol and diazepam in anxious individuals: An evaluation of the self-medication hypothesis. Psychopharmacology, 121, 91-103. doi: 0.1007/ BF02245595

Clevenger, T. J., \& Phifer, G. (1959). What do beginning college speech texts say about stage fright? The Speech Teacher, 8(1), 1-10. doi: 10.1080/03634525909376987

Cody, M. W., \& Teachman, B. A. (2011). Global and local evaluations of public speaking performance in social anxiety. Behavior Therapy, 42(4), 601-611. doi: 10.1016/j.beth.2011.01.004

Del Prette, A., \& Del Prette Z. A. P. (2001a). Psicologia das relaçôes interpessoais: Vivências para o trabalho em grupo. Petrópolis, RJ: Vozes.

Del Prette, A., Del Prette, Z. A. P., \& Castelo Branco, U. V. (1992a). Competência social na formação do psicólogo. Paidéia: Cadernos de Psicologia e Educação, 2, 40-50. doi: 10.1590/S0103-863X1992000200005

Del Prette, Z. A. P., Del Prette, A., \& Castelo Branco, U. V. (1992b). Competência social: Um levantamento de situações críticas de interação para estudantes de psicologia. Anais do I Congresso Nacional de Psicologia Escolar. Campinas, SP, Brasil, p. 384387. Recuperado de http://betara.ufscar.br:8080/ pesquisa/rihs/armazenagem/pdf/artigos/ del-prette-z.-a.-p.-del-prette-a.-castelo-branco-u.-v.-1992-competencia-social-um-levantamento-de-situacoes-criticas-de-interacao-para-estudantes-de-psicologia

Del Prette, Z. A. P., Del Prette, A., \& Barreto, M. C. M. (1998). Análise de um inventário de habilidades sociais (IHS) em uma amostra de universitários. Psicologia: Teoria e Pesquisa, 14(3), 219-228. Recuperado de https://revistaptp.unb.br/index.php/ ptp/article/view/1507/468

Del Prette, Z.A.P., \& Del Prette, A. (2001b). Inventário de Habilidades Sociais (IHS-Del-Prette): Manual de aplicação, apuração e interpretação. São Paulo: Casa do Psicólogo.

Gilles, D. M., Turk, C. L., \& Fresco, D. M. (2006). Social anxiety, alcohol expectancies, and self-efficacy as predictors of heavy drinking in college students. Addictive Behaviors, 31(3), 388-398. doi: 10.1016/j. addbeh.2005.05.020

Goberman, A. M., Hughes, S., \& Haydock, T. (2011). Acoustic characteristics of public speaking: Anxiety and practice effects. Speech Communication, 53(6), 867-876. doi: 10.1016/j.specom.2011.02.005

Guimarães, F. S., Zuardi, A. W., \& Graeff, F. G. (1988). Effect of chlorimipramine and maprotiline on experimental anxiety in humans. 
Journal of Psychopharmacology, 1(3), 184-192. doi: 10.1177/026988118700100305

Harb, G. C., Eng, W., Zaider, T., \& Heimberg, R. G. (2003). Behavioral assessment of public-speaking anxiety using a modified version of the Social Performance Rating Scale. Behaviour Research and Therapy, 41(11), 1373-1380. doi: 10.1016/ S0005-7967(03)00158-X

Kim, H. Y., Lu-ndh, L. G., \& Harvey, A. (2002). The enhancement of video feedback by cognitive preparation in the treatment of social anxiety. A single-session experiment. Journal of Behavior Therapy and Experimental Psychiatry, 33(1), 19-37. doi: 10.1016/S0005-7916(02)00010-1

Landim, A. K. P., Costa, C. F., Conilheiro, D., Sá, E. S., Nunes, E.C., Santos, E. E., ... Martinez, A. (2000). Ansiedade social em estudantes universitários: Preocupações mais emergentes. Revista Unicastelo, 3(3), 185-192.

Laukka, P., Linnman, C., Åhs, F., Pissiota, A., Frans, Ö., Faria, V., \& Furmark, T. (2008). In a nervous voice: Acoustic analysis and perception of anxiety in social phobics' speech. Journal of Nonverbal Behavior, 32(4), 195-214. doi: 10.1007/ s10919-008-0055-9

Levitan, M. N., Falcone, E. M., Placido, M., Krieger, S., Pinheiro, L., Crippa, J. A., \& Nardi, A. E. (2012). Public speaking in social phobia: A pilot study of self-ratings and observers' ratings of social skills. Journal of Clinical Psychology, 68(4), 397-402. doi: 10.1002/jclp.20868

Marinho, A. C. F., Medeiros, A. M. de, Gama, A. C. C., \& Teixeira, L. (2017). Fear of public speaking: Perception of college students and correlates. Journal of Voice. 31 (1), doi: 10.1016/j.jvoice.2015.12.012

McNair, D. M., Frankenthaler, L. M., Czerlinsky, T., White, T. W., Sasson, S., \& Fisher, S. (1982). Simulated public speaking as a model of clinical anxiety. Psychopharmacology, 77(1), 7-10. doi: 10.1007/ BF00436092

Oliveira, M. A., \& Duarte, Â. M. M. D. (2004). Controle de respostas de ansiedade em universitários em situações de exposições orais. Revista Brasileira de Terapia Comportamental e Cognitiva, 6(2), 183-199. Recuperado de http://www.usp.br/rbtcc/index. $\mathrm{php} / \mathrm{RBTCC} /$ article/view/56/45
Osório, F. de L., Crippa, J. A.S., \& Loureiro, S. R. (2012). Aspectos cognitivos do falar em público: Validação de uma escala de autoavaliação para universitários brasileiros. Revista de Psiquiatria Clínica., 39(2), 4853. doi: 10.1590/S0101-60832012000200002

Osório, F. L., Crippa, J. A. S., \& Loureiro, R. S. (2008). Escala para autoavaliação ao falar em público (SSPS): Adaptação transcultural e consistência interna da versão brasileira. Revista de Psiquiatria Clínica., 35(6), 207-11. doi: 10.1590/S0101-60832009000100002

Palma, S. M., Guimarães, F. S., \& Zuardi, A.W. (1994). Anxiety induced by simulated public speaking and stroop color word test in healthy subjects: Effects of different trait-anxiety levels. Brazilian Journal of Medical Biological Research, 27, 2895-2902.

Puteri, N. D., \& Fakhrurrozi, M (2007). The relationship between positive mindset and anxiety in public speaking of students. Undergraduate Program, Faculty of Psychology - Gunadarma University, Indonesia. Recuperado de http://papers. gunadarma.ac.id/files/journals/5/articles/2527/ public/2527-7105-1- PB.pdf

Rapee, R. M., \& Abbott, M. J. (2007). Modelling relationships between cognitive variables during and following public speaking in participants with social phobia. Behaviour Research and Therapy, 45(12), 2977-2989. doi: 10.1016/j.brat.2007.08.008

Schulz, S. M., Alpers, G. W., \& Hofmann, S. G. (2008). Negative self-focused cognitions mediate the effect of trait social anxiety on state anxiety. Behaviour Research and Therapy, 46(4), 438-449. doi: 10.1016/j. brat.2008.01.008

Thompson, S., \& Rapee, R. M. (2002). The effect of situational structure on the social performance of socially anxious and non-anxious participants. Journal of Behavior Therapy and Experimental Psychiatry, 33(2), 91-102. doi: 10.1016/ S0005-7916(02)00021-6

Voncken, M. J., \& Bögels, S. M. (2008). Social performance deficits in social anxiety disorder: Reality during conversation and biased perception during speech. Journal of Anxiety Disorders, 22(8), 13841392. doi: 10.1016/j.janxdis.2008.02.001

Wenzel, A., \& Holt, C. S. (2003). Social-evaluative threat and cognitive performance in socially anxious and nonanxious individuals. Personality and 
Individual Differences, 34(2), 283-294. doi: 10.1016/

Recebido em: 05/09/2016

S0191-8869(02)00044-2

Reformulado em: 24/04/2017

Zimbardo, P. G. (1982). A Timidez: Lisboa: Edições70.

Aprovado em: 04/08/2017

Nota dos autores:

Pesquisa parcialmente financiada pela Fundação de Amparo à Pesquisa do Estado de Minas Gerais (FAPEMIG - Processo n'. APQ-04059-10)".

Sobre os autores:

Antonio Paulo Angélico é professor adjunto do Departamento de Psicologia da Universidade Federal de São João del-Rei, doutor em Saúde Mental pela Faculdade de Medicina de Ribeirão Preto da Universidade de São Paulo e mestre em Educação Especial pela Universidade Federal de São Carlos.

E-mail: angelico.fmrp.usp@gmail.com

Murilo Freitas Bauth é psicólogo graduado pelo Centro Universitário de Lavras (UNILAVRAS) é mestre em Psicologia (Instituições, Saúde e Sociedade) pelo Programa de Pós-Graduação em Psicologia da Universidade Federal de São João del-Rei (UFSJ).

E-mail: murilobauth.psi@gmail.com

Arthur Kelles Andrade é psicólogo graduado pela Universidade Federal de São João del-Rei (UFSJ), atuou no Laboratório de Pesquisa em Saúde Mental (LAPSAM) da UFSJ, durante quatro anos, como estagiário em pesquisa e bolsista de iniciação científica.

E-mail: arthur.kelles@gmail.com

Contato com os autores:

Professor Doutor Antonio Paulo Angélico

Praça Dom Helvécio, 74, Departamento de Psicologia, Dom Bosco

São João del-Rei - MG, Brasil

CEP: 36301-160

Psico-USF, Bragança Paulista, v. 23, n. 2, p. 347-359, abr./jun. 2018 


\section{ERRATA 2}

No artigo "Estudo Experimental do Falar em Público Com e Sem Plateia em Universitários", com número de DOI: 10.1590/1413-82712018230213, publicado no periódico Psico-USF, 23(2): 347-359, na página 350, o trecho:

"Para esta última subescala, a pontuação dos itens é invertida, ou seja, quanto menor a média da pontuação, maior a avaliação negativa que o indivíduo tem de si (Osório et al., 2008)."

que estava alocado no $4^{\circ}$ parágrafo da referida página (imediatamente antes da seção Procedimentos) foi realocado para o $1^{\circ}$ parágrafo da mesma página (imediatamente antes da descrição do instrumento Teste de Simulação de Falar em Público). 


\section{ERRATA 3}

No artigo "Estudo Experimental do Falar em Público Com e Sem Plateia em Universitários”, com número de DOI: 10.1590/1413-82712018230213, publicado no periódico Psico-USF, 23(2): 347-359, nas páginas 347 e 359:

Onde se lia: "Arthur Keller Andrade"

Leia-se: "Arthur Kelles Andrade" 